\title{
Abstract \\ The next step: Video laryngoscopy with extended functions
}

Herath RMHMAI ${ }^{1}$

${ }^{1}$ National Hospital Sri Lanka

Key words: laryngoscopy, endotracheal intubation

\section{Introduction}

Direct laryngoscopy remains to be the method of choice in endo-tracheal intubation. However, it is difficult to perform direct laryngoscopy in $1-4 \%$ of the patients. Devices are being invented to facilitate the laryngoscopy and intubation where video laryngoscopes play a key role. Most of these devices demand reasonable mouth opening and they occupy most of the oral cavity - limiting the space to introduce the endotracheal tube (ETT). Even though they facilitate visualization of Larynx, they do not aid passing the ETT through the glottis. This is mostly experienced when the larynx is situated much anteriorly.

Objective

To develop a low cost video laryngoscope that can be used in patients with minimal mouth opening and reduced neck extension

Methods

This novel video-laryngoscope blade is made of stainless steel with a unique curvature and uniform thickness throughout the length. It is incorporated with a flexible video camera with inbuilt light source. Separate ports for pre-loaded bougie and oxygen insufflation are extended features of this device. The handle is placed on the same curvature of the blade to facilitate laryngoscopy.

Results

This device has been used in 28 patients with anticipated difficult airways and 05 unanticipated airway emergencies where all patients were intubated successfully with no major airway trauma, delay or desaturation of SPO2 less than $96 \%$.

Conclusion

This device can be used as an alternative advanced laryngoscope in anticipated difficult airways as well as a rescue method in airway emergencies. Lightweight, maneuverability, durability and significantly low manufacturing cost of this novel model will make a huge difference in management of difficult airway situations.

Corresponding Author: RMHMAI Herath, Email:<nanasawila@gmail.com>

Presentation at the $5^{\text {th }}$ biennial academic sessions of the Postgraduate institute of Medicine on 06 October 2016

Competing Interests: Authors have declared that no competing interests exist. 